\title{
Performance of the Forward RICH Detector System at DELPHI
}

\section{W. Adam et al.}

\begin{abstract}
One quarter of the Forward Ring Imaging Cherenkov detector is installed and has been operated in the DELPHI experiment. The detector covers the forward-backward regions $\left(15^{\circ}<\theta<35^{\circ}\right)$. Two radiator systems are used for particle identification in the momentum range up to $40 \mathrm{GeV} / \mathrm{c}$, a liquid Perfluorohexane and the Perfluorobutane gas. UV-photons with wave lenghts from $\sim 170 \mathrm{~nm}$ to $\sim 200 \mathrm{~nm}(7.3-6.2 \mathrm{eV})$ are detected with high efficiency. The total active area of the photon detector is $\sim 8 \mathrm{~m}^{2}$.
\end{abstract}





\title{
Performance of the Forward RICH Detector System at DELPHI.
}

\author{
W.Adam ${ }^{1}$, E.Albrecht ${ }^{1}$, I.Ambec ${ }^{2}$, A.Augustinus ${ }^{3}$, C.Barnoux ${ }^{4}$, B.Boštjančič ${ }^{5}$, O.Botner ${ }^{2}$, A.Budziak ${ }^{6}$, \\ P.Carechio ${ }^{4}$, P.Cavalli ${ }^{4}$, L.Ceelie ${ }^{3}$, R.Cereseto ${ }^{7}$, G.Cerutti ${ }^{1}$, E.Dahl-Jensen ${ }^{8}$, Ph.Dam $^{8}$, G.Damgaard ${ }^{8}$, \\ N.Dimitriou ${ }^{9}$, W.Dulinski ${ }^{6 a}$, L.O.Eek ${ }^{2}$, T.Ekelöf ${ }^{2}$, J.Erikson ${ }^{2}$, A.Florek ${ }^{6}$, B.Florek ${ }^{6}$, F.Fontanelli ${ }^{7}$,

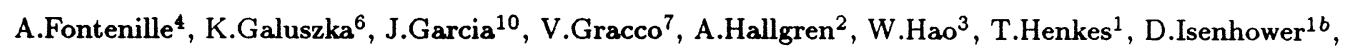 \\ H.Johansson ${ }^{2}$, E.Karvelas ${ }^{9}$, P.Kindblom ${ }^{2}$, B.Koene ${ }^{3}$, N.de Koning ${ }^{3}$, A.Korporaal ${ }^{3}$, P.Kostarakis ${ }^{9}$, \\ G.Lenzen $^{11}$, L-E.Lindqvist ${ }^{2}$, P.Lorenz ${ }^{11 c}$, D.Loukas ${ }^{9}$, B.Lund-Jensen ${ }^{2 d}$, A.Maltezos $^{9}$, A.Markou $^{9}$, \\ L.Mattsson $^{2}$, J.Medbo ${ }^{2}$, J.Michalowski ${ }^{6}$, F.Montano ${ }^{7}$, B.S.Nielsen ${ }^{8}$, K.Pakonski ${ }^{6}$, C.Perdikes ${ }^{9}$, G.Polok ${ }^{6}$, \\ A.Robohm ${ }^{1 e}$, G.Sajot ${ }^{4}$, M.Sannino ${ }^{7}$, E.Saragas ${ }^{9}$, E.Schyns ${ }^{11}$, S.Squarcia ${ }^{7}$, G.Stavropoulos ${ }^{9}$, M.Stodulski ${ }^{6}$, \\ Z.Stopa ${ }^{6}$, J.Thadome ${ }^{11}$, G.Theodosiou ${ }^{9}$, Z.Thomé ${ }^{12}$, L.Traspedini ${ }^{7}$, M.Turala ${ }^{6}$, O.Ullaland ${ }^{1}$, A.S.de la Vega ${ }^{12}$, \\ A.Wärm ${ }^{2}$, J.Werner ${ }^{11}$, S.Xyroutsikos ${ }^{9}$, M.Zavrtanik ${ }^{5}$ and E.Zevgolatakos ${ }^{9}$. \\ ${ }^{1}$ CERN, Geneva, Switzerland. ${ }^{2}$ Dept. of Rad. Sciences, Uni. of Uppsala, Uppsala, Sweden. \\ ${ }^{3}$ NIKHEF-H, Amsterdam, The Netherlands. ${ }^{4}$ Inst. des Sciences Nucléaires, Univ. de Grenoble, France. \\ ${ }^{5}$ Inst. Jozef Stefan, Ljubljana, Slovenija. ${ }^{6}$ Inst. of Nuclear Physics, Cracow, Poland. \\ ${ }^{7}$ Dip. di Fisica, Uni. of Genova and INFN, Italy. ${ }^{8}$ Niels Bohr Institute, Copenhagen, Denmark \\ ${ }^{9}$ NCSR Democritos, Athens, Greece. ${ }^{10}$ Uni. of Santander, Santander, Spain. \\ ${ }^{11}$ Fachbereich Physik, Uni. of Wuppertal, Wuppertal, FRG. ${ }^{12}$ COPPE, Rio de Janeiro, Brazil. \\ ${ }^{a}$ Present address: IN2P3-CNRS/ULP, Strasbourg, France. ${ }^{b}$ Visitor from Abiline Ch. Univ., Texas, USA. \\ ${ }^{c}$ Present address: Bayer Leverkusen, FRG. ${ }^{d}$ Present address: MSI, Stockholm, Sweden \\ ePresent address: Inst. für Exp. Kernphysik, Univ. Karlsruhe, Karlsruhe, FRG.
}

SUBMITTED TO IEEE NSS 1992

\section{Abstract}

One quarter of the Forward Ring Imaging Cherenkov detector is installed and has been operated in the DELPHI experiment. The detector covers the forward-backward regions $\left(15^{\circ}<\theta<35^{\circ}\right)$. Two radiator systems are used for particle identification in the momentum range up to $40 \mathrm{Gev} / \mathrm{c}$, a liquid Perfluorohexane and the Perfluorobutane gas. UV-photons with wave lengths from $\sim 170 \mathrm{~nm}$ to $\sim 200 \mathrm{~nm}(7.3-6.2 \mathrm{eV})$ are detected with high efficiency. The total active area of the photon detector is $\sim 8 \mathrm{~m}^{2}$.

\section{Introduction}

The Forward Ring Imaging CHerenkov detector (Forward RICH) [1] is part of the particle identification system of the DELPHI experiment (figure 1) [2] at the CERN LEP collider. It is used to do hadron identification over most of the momentum range up to $40 \mathrm{GeV} / \mathrm{c}$ by Cherenkov angle reconstruction. The detector covers the forward-backward region of the experiment.

In the first part of this paper we will give a description of the detector. Based on the detailed knowledge of the detection efficiency, the performance of the analog and digital electronics and the momentum reconstruction of the DELPHI detector, the measured resolution of the Forward
RICH is compared to simulation studies.

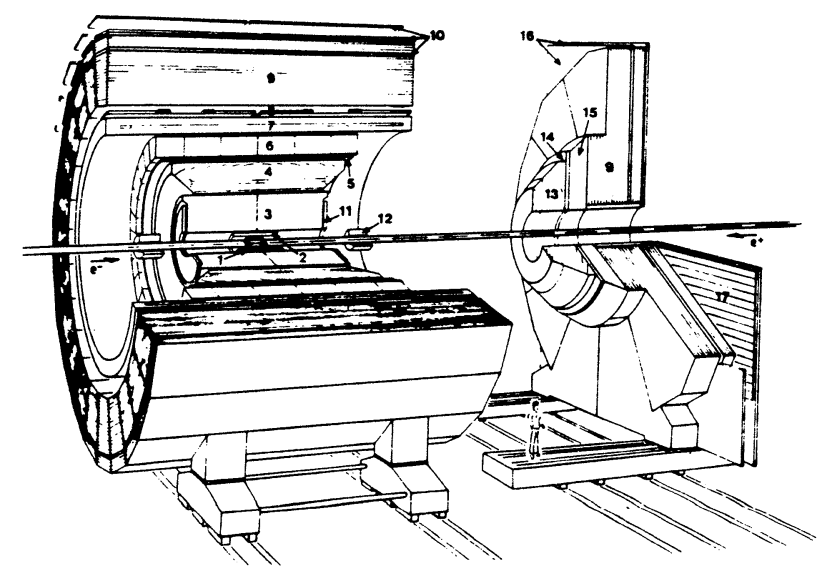

Figure 1: The DELPHI detector [2]. 13:Forward RICH, 11:chamber A, 14:chamber B.

\section{Detector Description}

Cherenkov photons in the UV range from both liquid and gaseous radiators are detected in a single array of photosensitive Time Projection Chambers (TPC). From the photon coordinates measured in the TPC plane, emission 
angles with respect to particle tracks are reconstructed. For a given particle momentum, mass hypotheses are tested against the observed number of photons and the distribution of Cherenkov angles of individual photons.

Fig. 2 shows the arrangement of the principal parts of the Forward RICH. One detector consists of two $180^{\circ} \mathrm{seg}$ ments, joined together in the vertical plane.

These are in turn assembled out of two parts:

1. The main vessel which houses the photon detectors and the mirrors. It serves as the gas radiator volume.

2. A shallower vessel is flanged onto the main vessel. It carries the liquid radiator containers.

The RICH counters are not stand-alone instruments. They rely on tracking detectors for the determination of particle momenta, as well as for the measurement of track coordinates. The Forward RICH is sandwiched between two tracking chambers (11 and 14 in fig. 1). Furthermore, the track detectors in the central region of DELPHI, notably the inner detector and the time projection chamber ( 2 and 3 in fig. 1), contribute to forward tracking down to $\theta \sim 20^{\circ}$.
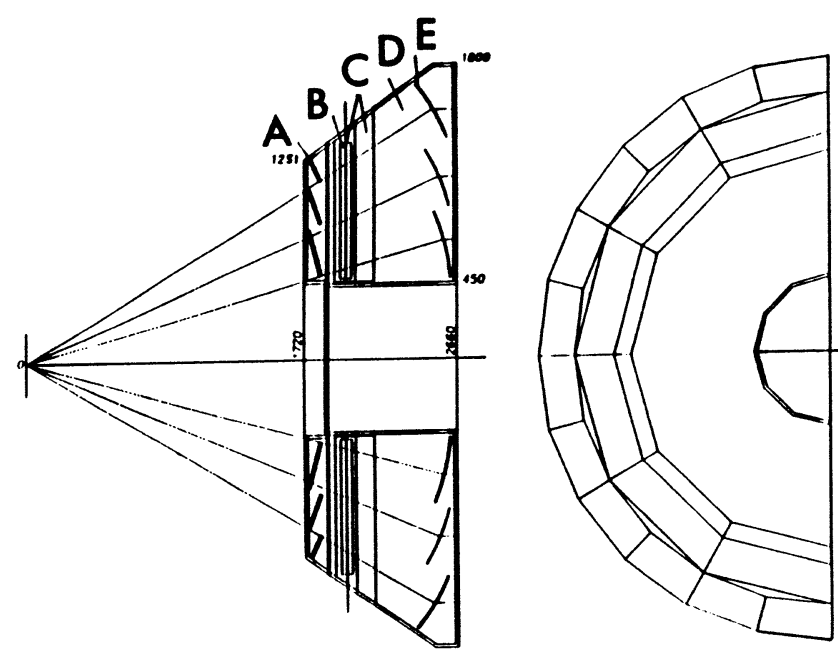

Figure 2: Schematic view the Forward RICH [1]. A:Liquid radiators, B:Photon detectors, D:Gas radiator, E:Mirrors.

\section{The Cherenkov Radiators}

\subsection{Liquid Radiator}

The liquid radiator plane in each end cap consists of 36 discrete containers filled with Perfluorohexane, $C_{6} F_{14}[3]$. The number of photons per particle obviously increases with the radiator thickness, but so does the uncertainty on their emission points. The combined effect is that the error on the Cherenkov angle is minimized when the radiator thickness is around $1 \mathrm{~cm}$. The containers are mounted inside two semi-circular vessels that are flanged onto the main vessel structure (see fig. 2). The acceptance in polar angle is between $16.3^{\circ}$ and $34.6^{\circ}$. The adopted geometry with the radiator planes approximately perpendicular to the particle track, minimizes refraction of the Cherenkov light. The Cherenkov image on the detection plane therefore occupies the smallest possible fiducial area. This optimizes the signal to background ratio.

The exit windows of the containers consist of UV-grade fused silica plates [4] of $5.0 \mathrm{~mm}$ thickness. All other parts of the liquid radiator structure are constructed out of a composite material of carbon fiber and epoxy. Each container is served by its own input capillary. The return line consists of a common sewer.

Oxygen and water is taken out of the liquid before it is pushed with an overpressure of $\sim 2$ bar from the distribution manifolds to the liquid containers via the $30 \mathrm{~m}$ long capillary tubes. Pressure sensors are installed on all input lines, 1-4 $\mathrm{m}$ away from the radiators. Samples of the liquid can be checked for UV transparency with a monochromator before and after cleaning.

We have observed this year traces of uncompleted fluorination of the hydrocarbons. As a consequence the liquid could not be de-oxygenized with our standard catalyst $(\mathrm{Cu}$ or $\mathrm{Cr}$ ). The oxygen has therefore been taken out by bubbling Argon through the liquid. The water is trapped with molecular sieve [5]. The integrated transparency measured from 160 to $220 \mathrm{~nm}$ for a $1 \mathrm{~cm}$ long cell, is $\sim 88 \%$ of that of a pure liquid.

\subsection{The Gas Radiator}

Two identical systems provide Perfluorobutane, $C_{4} F_{10}[6]$, to the four $180^{\circ}$ segments of the Forward RICH. Nitrogen is used as a pressure stabilizing gas. The pressure in the gas radiator volume is referred to atmospheric pressure and is controlled to about $\pm 1 \mathrm{hPa}$. The gas purity is maintained by permanent flow through traps filled with a molecular sieve. Typical contaminations are $\leq 10 \mathrm{ppm}$ of $\mathrm{O}_{2}$ and $\leq 100 \mathrm{ppm}$ of $\mathrm{H}_{2} \mathrm{O}$ for the output gas. Ultraviolet transparency is measured with a monochromator. We find that the transparency for this gas is $\sim 100 \%$ from $162 \mathrm{~nm}$ upward. This gives an integrated transparency measured from 160 to $220 \mathrm{~nm}$ for a $15 \mathrm{~cm}$ cell, of $\sim 95 \%$ of that of a pure gas.

The spherical mirrors have full coverage in azimuth and cover the polar interval $12^{\circ}<\theta<35^{\circ}$. There are 60 mirrors in each end cap with 5 mirrors in each $30^{\circ}$ sector as shown in fig. 2. All mirrors have a radius of curvature of $120 \mathrm{~cm}$, with the axis pointing towards the interaction point.

The design is a compromise : On the one hand a small mirror size is preferred in order to maximize the track length in the gas radiator and because the Cherenkov photons produced by off-axis particles do not form perfect circles in the focal plane. The errors from this deviation, which will increase with mirror size, must be small compared to the measurement errors. On the other hand small mirrors mean that frequently photons from one particle are reflected by more than one mirror making the reconstruction more difficult, and that more light is lost in cracks between the mirrors. 
The mirrors are tilted by a few degrees in order to avoid shadowing by the boxes housing the MultiWire Proportional Chambers(MWPC) preamplifiers (section 4.2 and 6.1) and to avoid imaging into the inactive area due to Lorentz angle (section 4.1).

The reflectivity of each mirror was measured over the range $140-280 \mathrm{~nm}$ [7]. The average reflectivity at $180 \mathrm{~nm}$ is $87 \%$. The angular dispersion of each mirror has been measured by using a point source placed in the center of curvature of the mirror. The light intensity has fallen to $4 \%$ for an angular dispersion of $\pm 0.38 \mathrm{mrad}$.

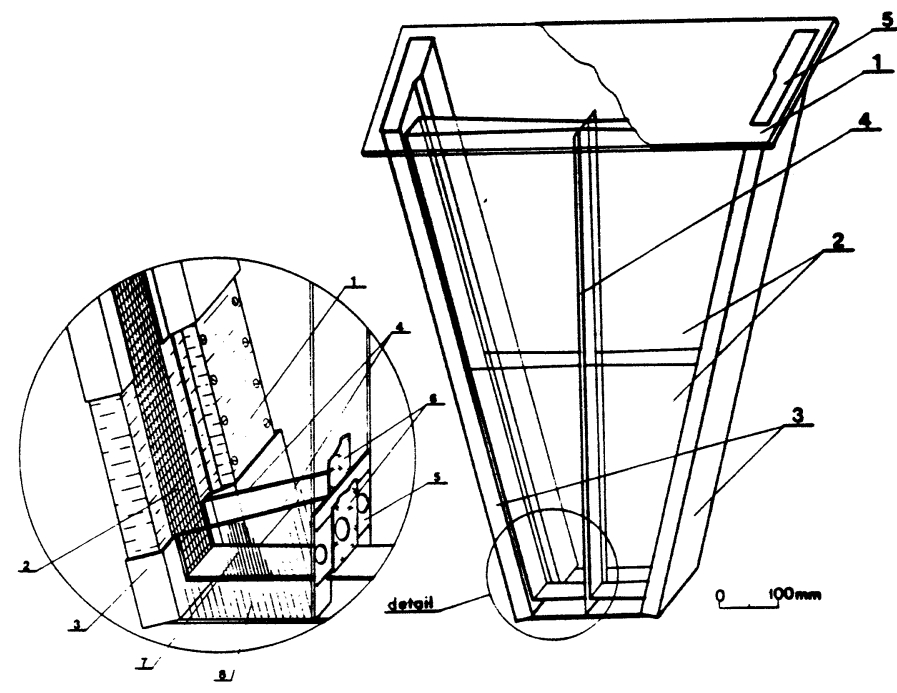

Figure 3: Drift box layout.

1:Support flange, 2:Quartz, 3:MWPC pocket, 4:Mid wall, 5:MWPC entrance.

In detail ; 1:Preamplifier housing, 2:MWPC, 3:MWPC pocket, 4:Quartz, 5:Mid wall, 6:Voltage interpolator, 7:Metallic strips, 8:Field wires.

\section{The Photon Detection}

\subsection{The Drift Boxes}

The photon detection plane is subdivided into trapezoidal TPC units. One unit, or drift box, covers a sector of $30^{\circ}$ (fig. 3). The drift volume is electrically divided into two symmetrical halves, each read out by a MWPC (section 4.2). The electric field of $1 \mathrm{kV} / \mathrm{cm}$ is perpendicular onto the chamber planes. The two sides of the drift box facing the liquid radiators and mirrors are made of UV grade fused quartz plates [4]. The supporting structure consists of glass fiber reinforced epoxy material (G10). The depth of the drift volume varies from $60 \mathrm{~mm}$ at the MWPCs to $40-54 \mathrm{~mm}$ along the mid wall. The MWPCs are housed in compartments ('pockets') made of a sandwich of glass fiber tissue and $2 \mathrm{~mm}$ of foam. The walls of the pocket are lined with Kapton foil to reduce outgassing.

The drift field is established by vacuum deposited metallic strips, $150 \mu \mathrm{m}$ wide at $3.0 \mathrm{~mm}$ pitch, on both sides of the quartz plates. Metallic strips printed on the end plates are connected to the window strips. Field shaping wires are placed about $25 \mathrm{~mm}$ outside the quartz windows. They provide further shielding of the drift field from external distortions. The field wire spacing is $9 \mathrm{~mm}$. In order to avoid excessive electric fields around these wires, their diameter varies from $50 \mu \mathrm{m}$ in the low field region to $200 \mu \mathrm{m}$ in the high field region. Strip and wire voltages are supplied by a resistor chain. One of the end plates serves as fixation of the drift box to the vessel.

The inherent inhomogeneity of the drift field around the mid wall of the detector is solved by using an ohmic glass voltage interpolator [8]. We have measured the drift distortions by mapping the drift volume using a fine grid single photo-electron source. All distortions are well below the uncertainty expected from diffusion in the drift gas. We observe no significant effect from accumulation of space charge or charging up of the glass and the quartz surfaces even with a large amount of charge deposited in the drift volume [9].

All quartz plates have been tested for UV transparency. The $50 \%$ transparency point is typically between 165 and $170 \mathrm{~nm}[10]$.

The 1.2 Tesla magnetic field of DELPHI is perpendicular to the electric field of the drift boxes. A fast drift gas implies a larger Lorentz angle. This influences the acceptance for Cherenkov photons from the liquid radiator. The photons produced in the gas radiator are less affected since the mirrors focus the light away from inactive regions (section 3.2). Charges must be drifted out of the volume within $10 \mu$ s due to the readout multiplexing scheme (section 6.1). The choice of drift gas is therefore a compromise between the requirements of a small Lorentz angle, a sufficiently high drift velocity and low diffusion. The gas must also have a good UV-transmission and be able to sustain high gas amplification. We have chosen Ethane $\left(\mathrm{C}_{2} \mathrm{H}_{6}\right)$. Fig. 4 shows the relationship between Lorentz angle and drift velocity as a function of the electric field for $B=1.2$ Tesla [11].

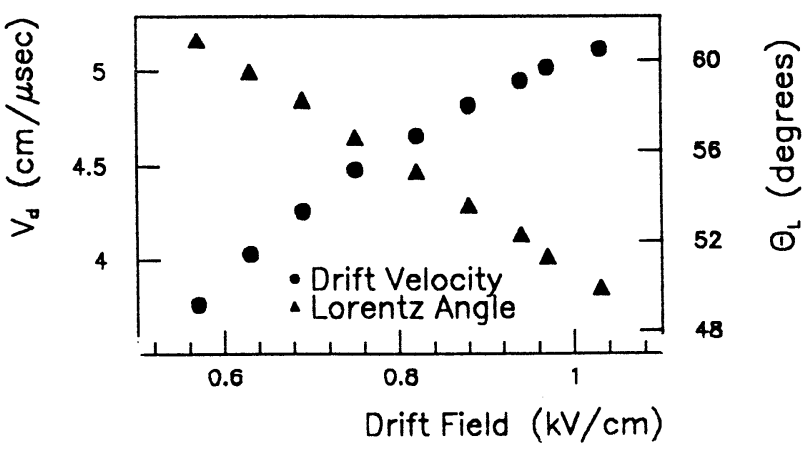

Figure 4: Lorentz angle and drift velocity versus electric field at $\mathrm{B}=1.2$ Tesla. 1992 data.

The shape of the drift boxes suppresses diffusion losses of charges drifting near the quartz windows as well as effects from inhomogeneity of the drift field, since the depth 
of the drift volume increases from the mid wall to the MWPCs (see fig. 3). This shape also increases the mechanical stability of the drift box.

The conversion of UV Cherenkov photons to free electrons is done by adding a photo-ionizing vapor to the drift gas. We use $\sim 0.6 \mathrm{hPa}$ of TMAE $\left(\mathrm{C}_{2}\left(\left(\mathrm{CH}_{3}\right)_{2} \mathrm{~N}\right)_{4}\right)$ in Ethane. With this TMAE concentration the photon mean free path is about $20 \mathrm{~mm}$.

\subsection{The MultiWire Proportional Cham- bers}

The MultiWire Proportional Chamber (MWPC), together with the electronics (section 6), detects and localizes each of the single photo-electrons in the Cherenkov image. A high detection efficiency is required for single photo electrons in the presence of ionizing tracks. The required detection efficiency is achieved by operating the chambers with a gas amplification $>10^{5}$. The anode wire channel, cathode pad channel and drift time information of the recorded signals give respectively the $r, z$, and $r \phi$ coordinates.

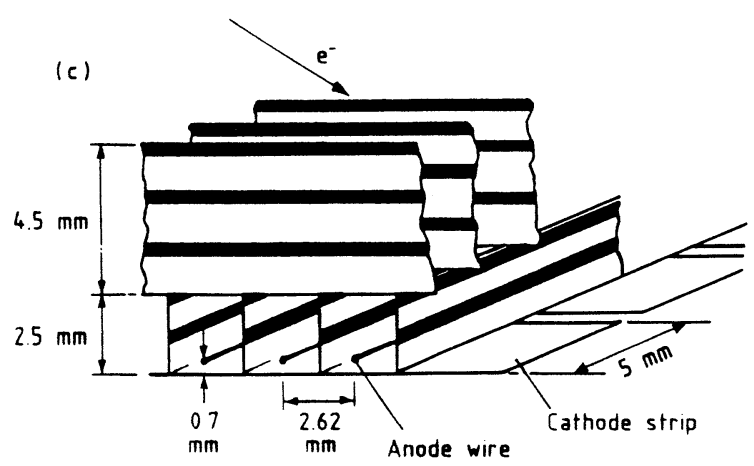

Figure 5: The proportional chamber.

The design is optimized for suppression of background signals from photons created in the avalanche around the anode wire (feed-back photo-electrons), to minimize crosstalk and to permit operation in a $1.2 \mathrm{~T}$ magnetic field. The high degree of crosstalk suppression needed follows from the large dynamic range between the lowest amplitude of the photo-electron signals and the very large signal amplitudes that are due to charged particles traversing the drift volume.

A cut through the MWPC is shown in fig. 5. Functionally the MWPC is divided into two parts, the detection plane and the preamplifier compartment. The detection plane of the MWPC has 320 anode wires spaced by $2.62 \mathrm{~mm}$ and 240 cathode pads. The cathode pads are arranged in 20 groups of 12 pads, each group being covered by 16 anode wires. The effective detection area is $841 \times 60 \mathrm{~mm}^{2}$. The height of the anode wire above the cathode plane is $0.7 \mathrm{~mm}$.

Feed-back photo-electron signals are limited by the optical screening structure. This structure is built from two crossed layers of precision-cut screens. It removes about $80 \%$ of the generated feed-back photons and permits lossfree photo-electron transfer with a fairly low electrical field applied over the structure, also when the $1.2 \mathrm{~T}$ magnetic field is applied parallel to the anode wires. Crosstalk signals due to pulses created on the electrodes of the screens are reduced by decoupling of the electrodes on the lower screens.

A suitable operating voltage for the anode wires has been determined in tests with single photo-electrons, liberated through ionization induced by UV-light from a hydrogen flash lamp.

\section{The Calibration System}

The calibration system is shown in Fig 6 . UV light from a lamp is fed into 22 quartz fibers of $100 \mu \mathrm{m}$ diameter. They project collimated light on the photon detectors and allow the determination of drift velocity and Lorentz angle. Two alignment fibers are placed near each MWPC. They are used as fiducial marks. The relative position with respect to the photon detector of each light spot is measured with $\sim 30 \mu \mathrm{m}$ precision.

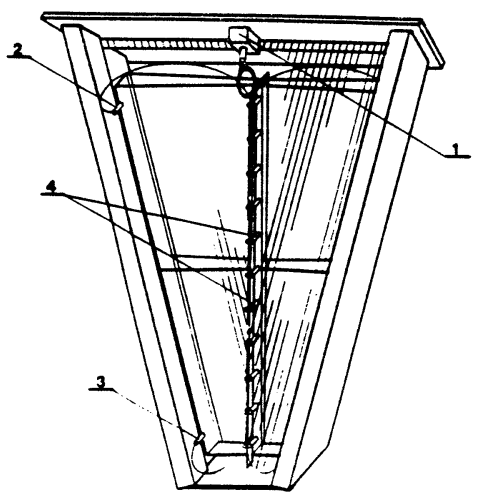

Figure 6: The UV calibration system. 1:UV lamp, 2-3:alignment fibers, 4:pointing fibers.

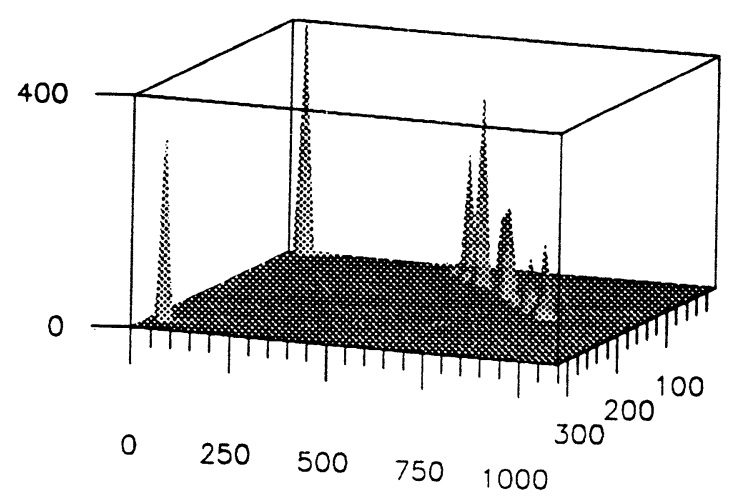

Figure 7: The UV calibration system. UV light spots as seen by one chamber.

The UV lamps are 3-window flash lamps developed especially for this system. They are Hydrogen filled lamps, 
free running at a frequency of $150 \mathrm{~Hz}$ when calibration data are taken.

Fig 7 displays a scatter plot of photons from the calibration system as seen with one MWPC. It shows the light spots near the mid wall of the drift volume, and the spots from the alignment fibers. Wire and time distributions of a spot, after matching in time the hits on wires and pads, have typical widths of $\sigma=0.85$ wires $(2.2 \mathrm{~mm})$ and $20 \mathrm{~ns}$ (1. $\mathrm{mm})$.

The drift velocities seen by all chambers are equal to within the statistical uncertainty, which is in the order of $0.2 \%$. The measured Lorentz angles agree with each other within $0.5 \%$. We expect that this agreement will improve when a more precise set of geometrical parameters will be used.

\section{Electronics and Data Acquisi- tion}

\subsection{The Electronics Chain}

The electronics chain [12] is designed to provide digitized time information for signals on each anode wire and cathode pad of the MWPCs. The high number of channels and severe space constraints inside the MWPC volume put strict requirements on simplicity of the circuits and cost effectiveness of the chain.

Track segments typically generate 600 primary electrons, distributed over about 10 wires. Single photoelectrons show a broad Polya distribution of pulse heights extending down to the electronic noise limit, while ionizing tracks have truncated Landau-like distributions.

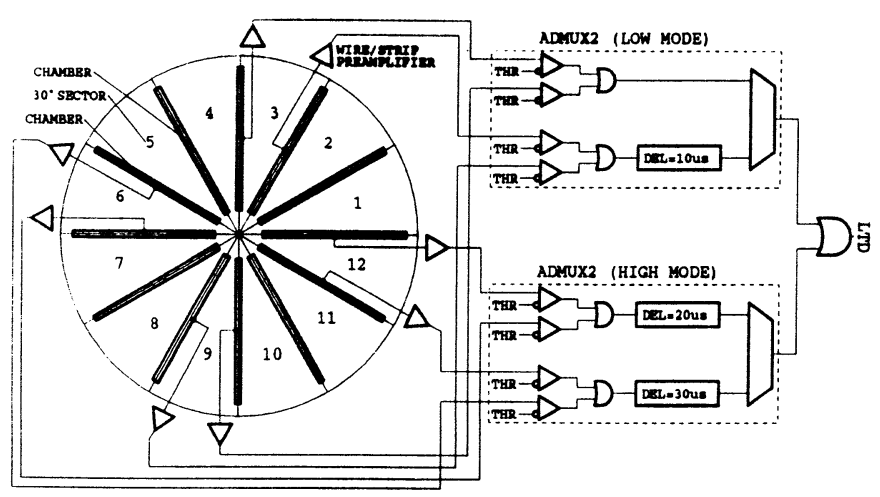

Figure 8: Elements of the electronics chain.

Figure 8 shows schematically the elements of the electronics chain. Preamplifiers are mounted directly in the MWPCs on each wire and strip. They are designed to detect the small single photo-electron signals, while sustaining the large signals from ionizing tracks with an acceptable dead-time. The total number of preamplifier channels is 26,880 .
The preamplifiers drive the signals through $40 \mathrm{~m}$ of shielded twisted pair cable to the rack-based electronics. The signals are received by Amplifier-DiscriminatorMUltipleXer (ADMUX2) Fastbus units. Each signal is amplified and discriminated against an adjustable threshold. Inside the ADMUX2 units, groups of discriminator outputs are delayed up to three times $10 \mu \mathrm{s}$ in order to achieve a time multiplexing of the signals at the output. They appear in four well-defined time slots, each $10 \mu \mathrm{s}$ long. This reduces the number of channels by a factor of four while retaining full acceptance over the $9 \mu$ s maximum drift time of the detector. Additional reduction by a factor of two is imposed by a direct ORing of the discriminated signals from two different chambers.

The LTD modules [13] complete the electronics chain. These Fastbus modules provide the time digitization relative to an external clock. A $100 \mathrm{MHz}$ clock burst provides a 10 ns time resolution. The same clock burst is used by the ADMUX2 modules to define the multiplexing delays and for the LTD time digitization. The start of the burst is synchronized to the LEP beam cross-over signal (BCO) through the local trigger supervisor unit, Pandora [14].

Space-points of single photon signals are formed off-line by combining corresponding wire and pad signals that coincide within \pm 30 ns. This time match strongly reduces the background to single photo-electron signals from electronic noise. By assuring in the cabling lay-out that matching wire and pad channels from one chamber are never ORed together in the same way as a matching pair from another chamber, the ambiguity introduced by the ORing can be fully resolved by the time match criterion.

\subsection{Data Acquisition}

The DELPHI data acquisition system is described in [2, 15]. The Forward RICH Fastbus system forms two partitions of this system, one for each end-cap. In addition to the LTD modules, each end-cap system contains a local trigger supervisor, Pandora, and a Fastbus Intersegment Processor (FIP) for readout control.

For accepted events, the data is stored in the four event deep front-end buffers in the LTD modules. The FIP is programmed to transfer the raw data from all LTD modules into its memory and format the event.

\section{Performance}

One quarter of the Forward RICH has been installed and has taken data for most of the running time at LEP during 1992. The rest of the detector will be installed during the winter shut-down 1992-93. The apparatus has proven to be very reliable and stable in operation. The TMAE temperature has been brought up to $25^{\circ} \mathrm{C}$, equivalent to a partial pressure of $0.57 \mathrm{hPa}$ or a conversion length for UV-photons of $19 \mathrm{~mm}$. It is clear from our operational experience, that the detector is very easy to operate since all the fluid systems are referred to atmospheric pressure, and 
the $\mathrm{C}_{4} \mathrm{~F}_{10}$ gas does not require the vessels to be heated.

Part of the data which we have until now, about $400 \mathrm{k} \mathrm{Z}^{0}$ events, have been compared to our Monte Carlo predictions [16]. See table 1. The difference between Cherenkov photons and reconstructed photons in table 1 , is mainly due to signals created by feed-back photons and ionizing tracks. The photons created in the quartz and the drift gas play a smaller role.

The uncertainty in the Cherenkov angle reconstruction will improve with better geometrical alignment and calibration constants. This work is in progress.

Table 1: Comparison between simulation and preliminary data for single $\mu$ events.

\begin{tabular}{|c|c|c|c|c|}
\hline Photons & \multicolumn{2}{|c|}{ Simulated } & \multicolumn{2}{c|}{ Data } \\
\hline & $\mathrm{C}_{6} \mathrm{~F}_{14}$ & $\mathrm{C}_{4} \mathrm{~F}_{10}$ & $\mathrm{C}_{6} \mathrm{~F}_{14}$ & $\mathrm{C}_{4} \mathrm{~F}_{10}$ \\
\hline Produced & 27 & 17.1 & & \\
\hline Detectable & 9.7 & 9.9 & & \\
\hline Reconstructed & 9.3 & 10.1 & & \\
\hline C-photons & 8.9 & 8.4 & $6.5 \pm .5$ & $7.0 \pm .3$ \\
\hline \hline $\begin{array}{c}\text { Uncertainty in } \\
\text { C-angle (mrad) }\end{array}$ & 9.0 & 2.8 & $11.5 \pm 1$ & $3.5 \pm .5$ \\
\hline
\end{tabular}

The particle identification power of the Forward RICH system suffers at the moment from insufficiency in tracking and momentum resolution below $\theta \sim 20^{\circ}$. This will be greatly improved with the upgrade of the DELPHI detector [17]. It is also expected that the Forward RICH TPC system will play an active role as a tracking detector. Preliminary results shows that it will have a resolution of $\pm 1 \mathrm{~mm}$.

\section{Conclusions}

The Forward RICH detector system is now operational in the DELPHI detector. Preliminary results for Cherenkov angle reconstruction show good agreement with simulated data. It will provide particle identification for hadrons from $\sim 2 \mathrm{GeV} / \mathrm{c}$ up to $\sim 40 \mathrm{GeV} / \mathrm{c}$. This opens up the possibility of an efficient study of the electro-weak couplings of the $\mathrm{Z}^{0}$ to various quark flavors by using leading kaons, protons and pions as a tag for primary $s, u$, and $d$ quarks.

\section{Acknowledgements}

We would like to thank our DELPHI collaborators for their vested interest in the project. The detector came into operation during physics data taking. A special thank to the online group for their patience during this start-up phase and to the technical support group for their invaluable help during the installation and the debugging of the detector. The dexterity with which C.Brand and his group solved the cooling of the preamplifiers and their work on the photon screens is gratefully acknowledged, as well as the skillful work of K.Ley on the recirculation system for the Cherenkov liquid. The work of S.Ilie on the fluorocarbons is gratefully acknowledged. P.Kokkinias is thanked for his effort in the slow control. We are indebted to F.Appelman for his work on running the fluid systems in 1990 and for the invaluable work of F.Formenti and his group in the electronics.

\section{References}

[1] O.Botner et al.; Forward RICH Review Report. DELPHI 88-7 GEN73/ RICH 30. 20 February 1988.

[2] P.Aarnio et al.; The DELPHI detector at LEP. (DELPHI Collaboration), Nucl.Instr.Meth. A303(1991)233.

[3] FC72 or PF5060. Product of 3M, Antwerpen.

[4] Nippon Silica Glass co.,LTD.

[5] Hydrosorb. Product of Messer Griesheim.

[6] DP L-12321. Product of 3M, St. Paul, Min., USA.

[7] P.Baillon et al.; An improved method for manufacturing accurate and cheap glass parabolic mirrors. Nucl.Instr.Meth. A276(1989)492

[8] Corning glass 0317 . The resistivity of the glass is about $10^{11} \Omega \mathrm{cm}$ at room temperature.

[9] B.Boštjančič et al.; Drift Optimization for the Photon Detectors of the DELPHI Forward Ring Imaging Detector. DELPHI 92-63 RICH 50, 10 June, 1992. Submitted to Nucl.Instr.Meth.A

[10] J.J.Garcia; Report on FRICH drift boxes transparency measurement. Forward RICH report April 19, 1991.

[11] W.Dulinski et al.; Operation of RICH single-photon detectors with optically shielded wires in transverse magnetic field. Nucl.Instr.Meth.A252(1986)418.

A.Markou et al.; Determination of drift velocity and Lorentz angle in the Forward RICH. DELPHI 91-95 RICH 45. 14 October 1991.

T.Kunst et al.; Precision measurement of magnetic deflection angles and drift velocity in crossed electric and magnetic fields. HD PY 92-08, Submitted to Nucl.Instr.Meth.A

[12] P.Dam et al.; Design and performance of the Readout Electronics Chain of the DELPHI Forward Ring Imaging Cherenkov Detector. IEEE NS 39(1992)

CERN ECP 91-32.

DELPHI 91-98 RICH 46 ELEC 36.

[13] G.Delavallade and J.P.Vanuxem; The LTD : a FASTBUS Time Digitizer for LEP experiments. Nucl.Instr.Meth. A252(1986)596.

[14] C.Quinton et al.; An Overview of the first and second level trigger in DELPHI. IEEE NS 36(1989)390

[15] W. Adam et al.; The DELPHI Fastbus Readout System. CHEP conference, Tsukuba, March 11-18 1991.

T. Adye et al.; Architecture and Performance of the DELPHI Data Acquisition and Control System, idem.

[16] E.Dahl-Jensen; Monte Carlo for the Forward RICH. Forward RICH note January 1992.

[17] The DELPHI collaboration; Proposal for the Upgrade of DELPHI in the Forward Region.

DELPHI 92-142-GEN-135 